\title{
Title page-
}

\section{Pitfalls in mitochondrial epigenetics}

Tina Pawar and Lars Eide*

Department of Medical Biochemistry, Institute of Clinical Medicine, University of Oslo

*Corresponding author:

Lars Eide, Dept. Medical Biochemistry, B1.3054, Rikshospitalet, Sognsvannsveien 20, 0027 Oslo,

Norway. Email: lars.eide@medisin.uio.no

Phone: +4723071062 


\begin{abstract}
5-methylcytosine $(5 \mathrm{mC})$ and 5-hydroxymethylcytosine $(5 \mathrm{hmC})$ are fingerprints of epigenetic modifications. These bases have been found in the mitochondrial DNA (mtDNA) and together with the discovery of mitochondrial localization of DNA methyltransferases (DNMTs), this implies that mtDNA is under epigenetic regulation.

However, the indirect methods hitherto used to assess mitochondrial $5 \mathrm{mC}$ and $5 \mathrm{hmC}$ require attention as they readily generate artificial signals that may lead to erroneous conclusions. Here, we demonstrate how three independent, frequently used methods to identify epigenetic modification of DNA readily generate false mtDNA epigenetic signals. The three methods were selective $5 \mathrm{mC} / 5 \mathrm{hmC}$-mediated inhibition of restriction enzymes, bisulfite conversion and $5 \mathrm{hmC}$ glucosylation-dependent immunocapture. Adequate controls for all methods are suggested.
\end{abstract}

\title{
Keywords
}

5mC: 5-methylcytosine, 5hmC: 5-hydroxymethylcytosine, mtDNA: mitochondrial DNA, epigenetics

\section{Introduction}

Genomic $5 \mathrm{mC}$ and $5 \mathrm{hmC}$ are well known by their ability to regulate gene expression during development, X-chromosome inactivation and mediating diseases including neurodegeneration (Guibert and Weber 2013), (Weichenhan and Plass 2013), (Sherwani and Khan 2015). Besides nuclear DNA, the mtDNA constitutes approximately $1 \%$ of the cellular DNA and may exist as several thousands of copies per cell. The early discovery of possible epigenetic modification of mtDNA spans more than 40 years back, where researchers found indications of DNMTs and $5 \mathrm{mC}$ in mitochondrial fractions (Vanyushin, Kiryanov et al. 
1971). More recent reports demonstrated the mitochondrial localization of DNMT isoforms as well as 5mC and 5hmC in mtDNA (Chen, Dzitoyeva et al. 2012), (Iacobazzi, Castegna et al. 2013), (Wong, Gertz et al. 2013), (Dzitoyeva, Chen et al. 2012), (Shock, Thakkar et al. 2011). The restriction enzymes HpaII and MspI have been used to quantify methylation, as the former is inhibited by $\mathrm{CpG}$ methylation in the CCGG recognition sequence, whereas MspI is not. This selective inhibition of HpaII over MspI underlies the estimated methylation frequency of $3-5 \%$ of the mitochondrial CpG sites (Pollack, Kasir et al. 1984). Epigenetic modification of mtDNA was later found to actively correlate with cellular homeostasis in neurons and Hela cells (Chestnut, Chang et al. 2011), (Shock, Thakkar et al. 2011). In these studies, the authors used antibody-based identification and immunoprecipitation of 5mC- and 5hmC containing DNA. The control region contains important binding sites for mtDNAbinding regulatory proteins. This region was found to be methylated in a non-CpG pattern, suggesting that $\mathrm{CpG}$ methylation is not a general requirement for epigenetic modification of the mtDNA. This finding was based on the identification of bisulfite-resistant cytosines (Bellizzi, D'Aquila et al. 2013). Intriguingly, a separate report showed that promotor regions of the central mitochondrial biogenesis regulator PGC-1 $\alpha$ in nuclear DNA was also subjected to non-CpG methylation (Barres, Osler et al. 2009), alluding to a distinct mode of epigenetic modification to regulate mitochondrial activity in the cell.

The multi-copy situation of mtDNA opens for individual use of cellular molecules. In view of the heterogeneous cellular role of mitochondria in ATP production, intracellular signalling and iron-sulphur biogenesis, epigenetic modification of an individual mtDNA molecule could hypothetically be a strategy to regulate the distinct metabolism of the specific mitochondrion in which it resides. Along these lines, epigenetic modification could also serve to select mtDNA for transcription or replication, and discriminate between damaged and nondamaged molecules. These possibilities sets some requirements to the resolution of the techniques that 
are chosen to investigate mitochondrial epigenetics: since mtDNA copy number may reach several thousand and epigenetic modifications potentially are restricted to only few molecules, it means that the methods have to be able to detect signals representing less than $1 \%$ of a given site.

Here, we present essential controls that need to be included to correct for artificial signals associated with three commonly used methods to assess mitochondrial epigenetics.

\section{Materials and methods}

\section{Mouse material}

Fresh brain tissue from 6 months old male mice (c57/B1/6) was used for isolation of mtDNA. The project was approved by the Norwegian Animal Research Authority and in accordance with the laws and regulations controlling experimental procedures in Norway and the European Union's Directive 86/609/EEC.

For isolation of total DNA, tissue was collected and stored for $24-48 \mathrm{~h}$ in RNAlater ${ }^{\circledR}$ at $4{ }^{\circ} \mathrm{C}$ before being homogenised and stored at $-80^{\circ} \mathrm{C}$. Total DNA was isolated using the DNeasy Blood \& Tissue Kit (Qiagen, Hilden, Germany) according to the manufacturer's description.

For isolation of mtDNA, brain mitochondria were first isolated by homogenization of fresh tissue in a mitochondrial isolation buffer, 1X MSHE buffer $(0.21 \mathrm{M}$ mannitol, $0.07 \mathrm{M}$ sucrose, $10 \mathrm{mM}$ Hepes (pH 7.4), 1 mM EDTA, 1 mM EGTA), using a Potter-Elvehjem homogenizer and subsequent purification by differential centrifugation as described previously (Croteau, ap Rhys et al. 1997, Halsne, Esbensen et al. 2012). The pelleted mitochondria were stored at $-80^{\circ} \mathrm{C}$ and mtDNA isolation was carried out in the same way as for total DNA. Isolated DNA was stored at $-20^{\circ} \mathrm{C}$. 


\section{Preparation of synthetic DNA}

DNA duplexes used for controls were amplified by PCR using BIOTAQ ${ }^{\mathrm{TM}}$ DNA Polymerase (Bioline, London, UK) and primers listed in Table 1. Standard PCR conditions: Initial denaturation at $94{ }^{\circ} \mathrm{C}$ for 5 minutes followed by 30 cycles of $94{ }^{\circ} \mathrm{C}$ for 30 seconds, $60{ }^{\circ} \mathrm{C}$ for 30 seconds, $72{ }^{\circ} \mathrm{C}$ for 30 seconds and final annealing at $72{ }^{\circ} \mathrm{C}$ for $5 \mathrm{~min}$. Annealing temperatures deviate for primers used to amplify bisulphite converted DNA only $\left(57^{\circ} \mathrm{C}\right)$. The elongation time was increased by 30 seconds per $500 \mathrm{bp}$ if the product exceeded $500 \mathrm{bp}$. The PCR product was separated by $1.8 \%$ Agarose gel electrophoresis and purified with illustra GFX PCR DNA and Gel Band Purification Kit (GE Healthcare, Cardiff, UK). The DNA concentration was determined by Epoch ${ }^{\mathrm{TM}}$ Microplate Spectrophotometer (BioTek,

Winooski, VT).

5mC-containing DNA was prepared by incubating a 150 bp PCR-product (obtained by $12 \mathrm{~S}$ for $+12 \mathrm{~S}$ rev primers) at $37^{\circ} \mathrm{C}$ for 1 hour in a reaction mixture with 10 units of $\mathrm{CpG}$ Methyltransferase M.SssI (New England Biolabs, Ipswich, MA), $160 \mu \mathrm{M}$ of Sadenosylmethionine (SAM) in NEBuffer 2. Methyltransferase was heat inactivated at $65^{\circ} \mathrm{C}$.

5hmC-containing DNA was generated by PCR-amplification with 5-Hydroxymethylcytosine dNTP Mix (Zymo Research, Irvine, CA) and either ND1.482bp for and ND1.482bp rev or COX1.501bp for and COX1.501bp rev to obtain a 482 bp PCR-product and 501 bp PCRproduct, respectively. Negative controls were obtained with normal dNTP mix and same primers.

\section{Real-time qPCR quantification of DNA.}

Samples (1.8 ng DNA) were mixed with Power SYBR Green PCR Master Mix (Life Technologies, Carlsbad, CA). Applied Biosystems StepOnePlus ${ }^{\mathrm{TM}}$ RealTime PCR System (Applied Biosystems, Waltham, MA) was used for qPCR analysis with the following 
conditions: Initial denaturation at $95^{\circ} \mathrm{C}$ for 10 minutes followed by 40 cycles of $95{ }^{\circ} \mathrm{C}$ for 15 seconds and $60{ }^{\circ} \mathrm{C}$ for 1 minute. The difference in cycle threshold $(\Delta \mathrm{Ct})$ was used to quantify the linear difference, using the following formulation: $2^{\Delta \mathrm{Ct}}$. For comparing the quantity of fragments amplified by different primers, we corrected for the difference in primer efficiency, using the algorithm established by Pfaffl (Pfaffl 2001).

\section{Epigenetic methods}

Selective inhibition of HpaII over MspI restriction cleavage:

Unless otherwise stated, $60 \mathrm{ng}$ of DNA was digested with 10 units of either HpaII or MspI (New England Biolabs) in $1 \mathrm{X}$ CutSmart Buffer at $37^{\circ} \mathrm{C}$ for 1 hour and heat inactivated at $80^{\circ} \mathrm{C}$ for 30 minutes. Alternatively, NEBuffer 1 was used.

Selective inhibition of bisulfite-mediated deamination of cytosine to uracil:

Three different commercially available kits were evaluated, since the kits differ in efficiency (izzi (Izzi, Binder et al. 2014): Zymo Research EZ Methylation kit (Nordic BioSite, Täby, Sweden); EpiJET Bisulfite Conversion Kit (Waltham, MA) and the EpiTect Bisulphite Kit (Qiagen) were used for the bisulphite conversions according to the manufacturer's instructions. Where specified, linearization of DNA prior to conversion was achieved by digestion of $500 \mathrm{ng}$ of DNA with 20 units of BmtI HF (New England Biolabs) in 1X CutSmart Buffer at $37^{\circ} \mathrm{C}$ for 1 hour followed by heat inactivation at $65^{\circ} \mathrm{C}$ for 30 minutes. Linearization was confirmed by qPCR using Bmt-Ctr for and Bmt-Ctr rev primers, flanking one of the two sites for Bmt1 HF in mtDNA. The bisulphite converted DNA was amplified by PCR with primers specific to the converted mtDNA light $(\mathrm{L})$ and heavy $(\mathrm{H})$ strands $(\mathrm{BS}-12-\mathrm{L}$ for + rev and BS-COX1-H for + rev, respectively). The PCR products were purified, and sequenced by GATC Biotech Laboratories. Data analysis was carried out using the Chromas Lite 2.1.1 software from Technelysium (South Brisbane, Australia). 
$5 \mathrm{hmC}$ detection by glucosylation and immunoprecipitation of the resulting 5-glucosyl methylcytosine (5gmC) - containing DNA:

DNA to be analysed was fragmented by sonication with LABSONIC® M from Sartorius (Goettingen, Germany) (cycle 0.5, amplitude $30 \%$ ) for 30 seconds followed by 30 seconds on ice repeated in total 3 times, to yield an approximate fragment size of $500 \mathrm{bp}$, as verified by qPCR analysis with $12 \mathrm{~S} .494 \mathrm{bp}$ primers. Subsequent procedure was performed according to the manufactorer's description (The Quest 5-hmCTM DNA Enrichment kit from Zymo Research) with some modifications. Briefly, the fragmented DNA (1.5 $\mu \mathrm{g})$ was glucosylated by T4 Phage $\beta$-glucosyltransferase (New England Biolabs) in 5-hmC GT Reaction Buffer at $37^{\circ} \mathrm{C}$ for 1 hour before the addition of $500 \mu \mathrm{L}$ of JBP Binding Buffer and $10 \mu \mathrm{L}$ of JBP Capture MagBeads (Zymo Research). The reactions were incubated with end-over-end rotation at room temperature for 2 hours. The beads were then washed three times with JBP Binding Buffer before elution of 5hmC enriched DNA.

\section{Results and discussion}

We first evaluated the selective 5mC-mediated inhibition of CCGG cleavage by the restriction enzyme HpaII as compared to MspI. Positive control DNA was obtained by in vitro methylation of synthetic DNA containing a single CpG site (amplified by PCR) with M.SssI methyltransferase as described in materials and methods. The subsequent product was digested with MspI and HpaII and the remaining, intact DNA was quantified by real-time qPCR analyses. Methylated DNA efficiently inhibited HpaII (95\%) and strongly indicates complete methylation of the CpG substrate by M SSI methyltransferase. MspI cleavage was largely unaffected by methylation. In our experimental condition, only $5 \%$ of the original product escaped cleavage (Figure 1A). Unmethylated DNA served as control, and was similarly digested by both enzymes ( $3 \%$ uncut). We believe that the higher amount of uncut 
methylated compared to unmethylated DNA by MspI is due to DNA conformation changes induced by the in vitro methylation procedure, since 20 -fold dilution of DNA to increase the Enzyme : Substrate ratio did not result in more digestion (data not shown). Based on these results we find that the selective HpaII over MspI inhibition can be used to quantify internal $5 \mathrm{mC}$ methylation, but that artificial signals contribute from $3-5 \%$. Consequentially, this method has insufficient resolution to determine epigenetic signals lower than 5\%.

The efficacy of HpaII varied much more than that of MspI to various DNA preparations and recognition sites within a sequence (data not shown). To evaluate the potential sequencedependent variability in HpaII digestion, the mtDNA region spanning from nt 3241 to 3577 of $m t-N d 1$, containing two HpaII recognition sites was amplified by PCR. The purified PCR product was subjected to HpaII digestion using two commercially provided/recommended buffers. The efficacy of site-specific cleavage was then analysed for a given reaction condition. As shown in Figure 1B, the HpaII catalytic activity was sequence-dependent in a buffer-specific manner. Two recommended restriction buffers caused more than 50\% difference in enzymatic activity (compare left and right panels of Figure 1B). More importantly, while there was a clear difference in the digestion of the two sites by HpaII in one buffer, the enzyme worked equally and more efficient on both sites in another buffer. In the least favourable buffer, almost $10 \%$ of the sites resisted digestion even upon large excess of HpaII. Thus, the choice of commercially recommended buffers determines the efficiency of HpaII more than $50 \%$ and perhaps more unexpected, the sequence dependency of HpaII. In comparison, MspI activity was independent of site and equally efficient in both buffers. In conclusion, synthetic DNA (PCR amplified) covering the sequence of analyses must be included to control for sequence- and buffer specific inhibition of HpaII.

The gold standard for epigenetic analyses has been the investigation of bisulfite-treated DNA. Chemically, $5 \mathrm{mC}$ and $5 \mathrm{hmC}$ render single stranded DNA relatively resistant to bisulfite- 
mediated deamination, which otherwise converts unmodified cytosine to uracil in a subsequent desulfonation reaction. The method has been used to demonstrate epigenetic modification of mtDNA (Bellizzi, D'Aquila et al. 2013), (Pirola, Gianotti et al. 2013) One critical factor in the bisulfite conversion is the relative resistance of double stranded DNA to bisulfate-mediated cytosine deamination, which is usually overcome by a preceding denaturation step. Because of the plasmid nature of mtDNA, the denaturation is readily reversible unless the plasmid is linearized prior to the denaturation. (Frommer, McDonald et al. 1992) linearized plasmid DNA). The importance of this is demonstrated in Figure 2A, which demonstrates the apparent resistance to deamination of cytosines in the $m t$-CoxI gene that is dependent on preceding linearization of mtDNA. When using total DNA, it is a possibility that nuclear mitochondrial sequences (numts) can mask potential mtDNA-specific signals. However, as seen in Figure 2B, linearized DNA showed the same result, independent of whether total DNA or DNA from isolated mitochondria were used. We compared the presence of $12 \mathrm{~S}, \mathrm{Nd} 1$ and $\mathrm{Cox} 1$ sequences in total DNA with that obtained from PCR product covering all three, and found identical ratios, inferring that potential numts are unlikely to interfere with the results. Despite that bisulfite sequencing has been used to analyse mitochondrial epigenetics in several publications, we could only identify one report where the DNA was linearized prior to bisulfite conversion. Intriguingly, the authors of this work concluded that CpG methylation was absent in mtDNA (Hong, Okitsu et al. 2013). Linear mtDNA can also exist in cells (Linn (Higuchi and Linn 1995)) and can be formed during DNA isolation. To ensure complete linearization, it is recommended to introduce a separate restriction digestion step, which can be verified as shown in Figure 2.

The third principally different method used to analyse modified cytosines in the DNA, is antibody-based detection of $5 \mathrm{mC}$ or $5 \mathrm{hmC}$. Commercially available antibodies against $5 \mathrm{mC}$, 5hmC, 5-formylcytosine and 5-carbaxycytosine potentially allow for the discriminative 
quantification of these lesions, and are used in ELISA quantification kits or alternatively immunoprecipitation kits in preferential enrichment of epigenetically modified DNA fragments. $5 \mathrm{mhC}$ can be glucosylated to $5 \mathrm{gmC}$ by $\mathrm{T} 4 \beta$-glucosyltransferase (T4-BGT). The procedure was underlying the identification of very high levels of $5 \mathrm{hmC}$ in mouse embryonic stem cell mtDNA by a high throughput sequencing approach (Sun, Terragni et al. 2013). In view of the sequence specificity and DNA conformation dependency that were associated with the other techniques (Figures 1 and 2), we wanted to investigate possible sequencedependent variation in the $5 \mathrm{hmC}$-identification method as well.

A commercially available kit to quantify $5 \mathrm{hmC}$ by selective glucosylation of duplex-5hmC by T4-BGT, followed by fragmentation, binding, washing and subsequent elution from affinity beads (see materials and methods) was used. To omit supercoil interference, a $7 \mathrm{~kb}$ amplicon covering the mtDNA genes $m t-R n r l(12 \mathrm{~S})$, $m t-N d 1$ (ND1) and $m t$-Coxl (COX1) was amplified by PCR. The PCR product was fragmented according to manufacturer's protocol, and real-time qPCR was then employed to quantify the fragments. The yield of the three targets was similar from three independent DNA preparations (Figure 3A), demonstrating uniform fragmentation of the DNA. We incubated the fragmented, unmodified DNA with beads linked to $5 \mathrm{gmC}$ antibodies to test for potential variability in non-specific, sequencedependent affinity. To our surprise, we found that fragments containing $12 \mathrm{~S}$ and COX1sequences were retained 7-fold stronger than ND1-containing sequences on the beads (Figure 3B). The recovery of non-modified ND1 fragments yielded $10^{-6}$ of the input DNA. In comparison, 5hmC-positive DNA duplex generated by 5hmCTP incorporation was recovered at a yield of $10^{-4}$ of the specific input fragment, representing approximately 70 -fold preferential binding (Figure 3C). The similar enrichment of 5hmC-containing ND1 and COX1 fragments despite their different affinities to the beads shows the importance of relating $5 \mathrm{hmC}$ signals to that obtained from non-modified DNA with identical sequence. 
The identification of $5 \mathrm{hmC}$ by the $5 \mathrm{gmC}$ antibody requires a preceding glucosylation step by T4-BGT. Since unspecific glucosylation by T4-BGT would add another contribution to the artificial signal to $5 \mathrm{hmC}$, we tested for this possibility by incubating unmodified, PCRgenerated DNA with T4-BGT. Surprisingly, T4-BGT-treated DNA caused an increased "unspecific" binding to the affinity beads, varying more than 40 -fold. We did not find any correlation between the preferential antibody binding of specific sequences in Figure $3 \mathrm{~B}$ and their ability to be unspecifically glucosylated by T4-BGT (data not shown). Thus, the combined variability in unspecific binding and glucosylation therefore potentially can result in considerable spread of the $5 \mathrm{hmC}$ signals. To illustrate this, the individual $5 \mathrm{hmC}$ signals obtained from the $12 \mathrm{~S}$ and ND1 fragments prepared from either mouse brain mtDNA or synthetic, unmodified DNA were presented side by side in Figure 3D. The ND1 fragment contains $8 \mathrm{CpG}$ sites, compared to $1 \mathrm{CpG}$ in the $12 \mathrm{~S}$. Thus, it is not likely that the unequal affinity for non-modified DNA is due to the antibody recognizing intact $\mathrm{CpG}$ sites in the sequence. The signals were similarly spread for both types of DNA hence, it is crucial to correlate signals with that obtained from unmodified DNA. The 5-hydroxymethylome of mtDNA reported previously (Sun, Terragni et al. 2013) used the E.coli chromosome as a negative control. In view of our findings, a more suitable control would be the entire mtDNA sequence amplified by PCR.

In conclusion, false positive signals are readily obtained using commonly used tools to study epigenetic modifications of mtDNA. The artificial signals are either caused by unspecific activities of the enzymes used for detection, sequence variability or plasmid nature of mtDNA. Rather than indirect methods, identification of $5 \mathrm{mC}$ and other epigenetic markers by mass spectrometry has now shown to provide a unique tool to quantify the total genomic level of epigenetic markers. To our knowledge, there are hitherto no reports of mtDNA epigenetics 
based on mass spectrometry analyses. Thus, mass spectrometry identification of mtDNA epigenetic markers is needed to pursue mitochondrial epigenetics.

\section{Acknowledgements}

This study was supported by grants from the University of Oslo and Oslo University Hospital.

\section{Competing interests}

The authors declare no competing interests.

\section{References}

Barres, R., M. E. Osler, J. Yan, A. Rune, T. Fritz, K. Caidahl, A. Krook and J. R. Zierath (2009). "Non-CpG methylation of the PGC-1alpha promoter through DNMT3B controls mitochondrial density." Cell Metab 10(3): 189-198.

Bellizzi, D., P. D'Aquila, T. Scafone, M. Giordano, V. Riso, A. Riccio and G. Passarino (2013). "The control region of mitochondrial DNA shows an unusual CpG and non-CpG methylation pattern." DNA Res 20(6): 537-547.

Chen, H., S. Dzitoyeva and H. Manev (2012). "Effect of valproic acid on mitochondrial epigenetics." Eur J Pharmacol 690(1-3): 51-59.

Chestnut, B. A., Q. Chang, A. Price, C. Lesuisse, M. Wong and L. J. Martin (2011). "Epigenetic regulation of motor neuron cell death through DNA methylation." J Neurosci 31(46): 16619-16636.

Croteau, D. L., C. M. ap Rhys, E. K. Hudson, G. L. Dianov, R. G. Hansford and V. A. Bohr (1997). "An oxidative damage-specific endonuclease from rat liver mitochondria." J Biol Chem 272(43): 27338-27344.

Dzitoyeva, S., H. Chen and H. Manev (2012). "Effect of aging on 5-hydroxymethylcytosine in brain mitochondria." Neurobiol Aging 33(12): 2881-2891.

Frommer, M., L. E. McDonald, D. S. Millar, C. M. Collis, F. Watt, G. W. Grigg, P. L. Molloy and C. L. Paul (1992). "A genomic sequencing protocol that yields a positive display of 5methylcytosine residues in individual DNA strands." Proc Natl Acad Sci U S A 89(5): 18271831.

Guibert, S. and M. Weber (2013). "Functions of DNA methylation and hydroxymethylation in mammalian development." Curr Top Dev Biol 104: 47-83. 
Halsne, R., Y. Esbensen, W. Wang, K. Scheffler, R. Suganthan, M. Bjoras and L. Eide (2012). "Lack of the DNA glycosylases MYH and OGG1 in the cancer prone double mutant mouse does not increase mitochondrial DNA mutagenesis." DNA Repair (Amst) 11(3): 278285.

Higuchi, Y. and S. Linn (1995). "Purification of all forms of HeLa cell mitochondrial DNA and assessment of damage to it caused by hydrogen peroxide treatment of mitochondria or cells." J Biol Chem 270(14): 7950-7956.

Hong, E. E., C. Y. Okitsu, A. D. Smith and C. L. Hsieh (2013). "Regionally specific and genome-wide analyses conclusively demonstrate the absence of $\mathrm{CpG}$ methylation in human mitochondrial DNA." Mol Cell Biol 33(14): 2683-2690.

Iacobazzi, V., A. Castegna, V. Infantino and G. Andria (2013). "Mitochondrial DNA methylation as a next-generation biomarker and diagnostic tool." Mol Genet Metab 110(1-2): $25-34$.

Izzi, B., A. M. Binder and K. B. Michels (2014). "Pyrosequencing Evaluation of Widely Available Bisulfite Conversion Methods: Considerations for Application." Med Epigenet 2(1): 28-36.

Pfaffl, M. W. (2001). "A new mathematical model for relative quantification in real-time RTPCR." Nucleic Acids Res 29(9): e45.

Pirola, C. J., T. F. Gianotti, A. L. Burgueno, M. Rey-Funes, C. F. Loidl, P. Mallardi, J. S. Martino, G. O. Castano and S. Sookoian (2013). "Epigenetic modification of liver mitochondrial DNA is associated with histological severity of nonalcoholic fatty liver disease." Gut 62(9): 1356-1363.

Pollack, Y., J. Kasir, R. Shemer, S. Metzger and M. Szyf (1984). "Methylation pattern of mouse mitochondrial DNA." Nucleic Acids Res 12(12): 4811-4824.

Sherwani, S. I. and H. A. Khan (2015). "Role of 5-hydroxymethylcytosine in neurodegeneration." Gene.

Shock, L. S., P. V. Thakkar, E. J. Peterson, R. G. Moran and S. M. Taylor (2011). "DNA methyltransferase 1, cytosine methylation, and cytosine hydroxymethylation in mammalian mitochondria." Proc Natl Acad Sci U S A 108(9): 3630-3635.

Sun, Z., J. Terragni, J. G. Borgaro, Y. Liu, L. Yu, S. Guan, H. Wang, D. Sun, X. Cheng, Z. Zhu, S. Pradhan and Y. Zheng (2013). "High-resolution enzymatic mapping of genomic 5hydroxymethylcytosine in mouse embryonic stem cells." Cell Rep 3(2): 567-576.

Vanyushin, B. F., G. I. Kiryanov, I. B. Kudryashova and A. N. Belozersky (1971). "DNAmethylase in loach embryos (Misgurnus fossilis)." FEBS Lett 15(4): 313-316.

Weichenhan, D. and C. Plass (2013). "The evolving epigenome." Hum Mol Genet 22(R1): R1-6. 
Wong, M., B. Gertz, B. A. Chestnut and L. J. Martin (2013). "Mitochondrial DNMT3A and DNA methylation in skeletal muscle and CNS of transgenic mouse models of ALS." Front Cell Neurosci 7: 279. 


\section{FIGURE LEGENDS}

\section{Figure 1. Evaluating digestion of CpG-methylated DNA by restriction enzymes HpaII}

and MspI. (A) 5mC-containing DNA was prepared in vitro by methylating a purified, PCRamplified mtDNA fragment by M.SssI DNA methyltransferase, and subsequently analysing the respective sensitivity to HpaII and MspI restriction enzymes. The non-methylated mtDNA PCR-amplified DNA fragment was used as negative control. (B) Digestion of synthetic mtDNA fragment containing two HpaII sites were performed with increasing concentrations of enzyme and the site-specific efficacy of cleavage was compared in two different buffer conditions (left panel: CutSmart Buffer, right panel: NEBuffer 1). Synthetic DNA fragment was amplified using primers ND1.1 for and ND1.2 rev. Primer sets ND1.1 for + rev, and ND1.2 for + rev were used in qPCR to quantify cleavage of site 1 and 2, respectively. Results shown are obtained from three independent experiments, and presented as average with SD.

Figure 2. Sensitivity of mtDNA to bisulfite conversion. (A) Isolated total DNA was either treated with BmtI HF to linearize mtDNA or left nontreated (NT). The efficiency of BmtI-HF digestion was assessed by qPCR using primers flanking the BmtI-HF restriction site. (B) The NT DNA or BmtI-HF-treated DNA was subjected to bisulfite conversion using Zymo Research EZ Methylation kitdirectly prior to PCR-amplification and subsequent sequencing as indicated. The sequence shown is taken from mt-CoxI. Asteric indicates where cytosine (blue peaks) in NT DNA have been converted to uracil (thymine: red peaks) in the Bmt-HFtreated DNA. Two other kits gave identical results. (C) mtDNA from purified brain mitochondria was linearized, fragmented, bisulfite converted (using Qiagen Epitect kit) and subsequently sequenced. The top panel displays a selected region in the mt-Rnr1 sequence and the lower panel the same region obtained from total DNA as starting material. Asteric indicates positions where cytosines in the original sequence have been converted.The experiments were repeated at least three times with three independent DNA preparations. 


\section{Figure 3. Assessing accuracy of $5 \mathrm{hmC}$ quantification method including specificities of}

5gmC-antibody and glucosylation by T4-BGT. (A) Evaluation of the fragmentation of mtDNA. DNA was fragmented as described, and the relative yield of three fragments calculated from the CT values were determined from three separate DNA preparations and presented relative to the $12 \mathrm{~S}$ site (mean with SD are shown). (B) Sequence-dependent binding of unmodified DNA to affinity beads. Purified PCR product was subjected to $5 \mathrm{hmC}$ analyses as described, and the resulting recovery of the indicated fragments determined by qPCR and presented as yield of input $(* \mathrm{p}=0.03$; Student's t-test). (C) Purification of 5hmC-containing DNA. The enrichment of the indicated fragments were calculated as the preferential recovery of glucosylated compared to non-glucosylated 5hmC-containing fragments (D) Distribution of $5 \mathrm{hmC}$ signals obtained from $12 \mathrm{~S}$ (black) and ND1 (red) sequences, originated from mouse brain mtDNA $(\mathrm{n}=7)$ and glucosylated, 5hmC-free DNA. The recovered DNA is presented as yield of input. 
A

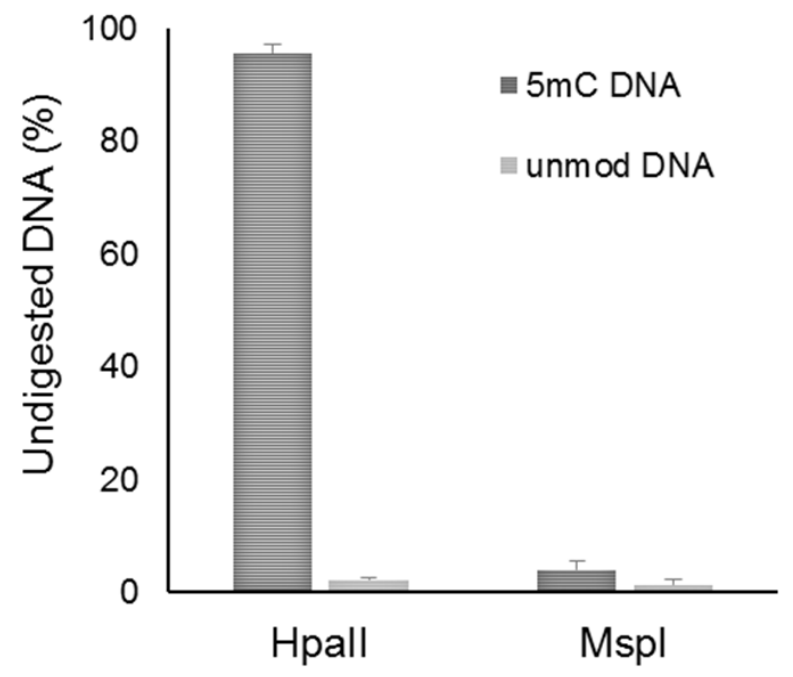

B
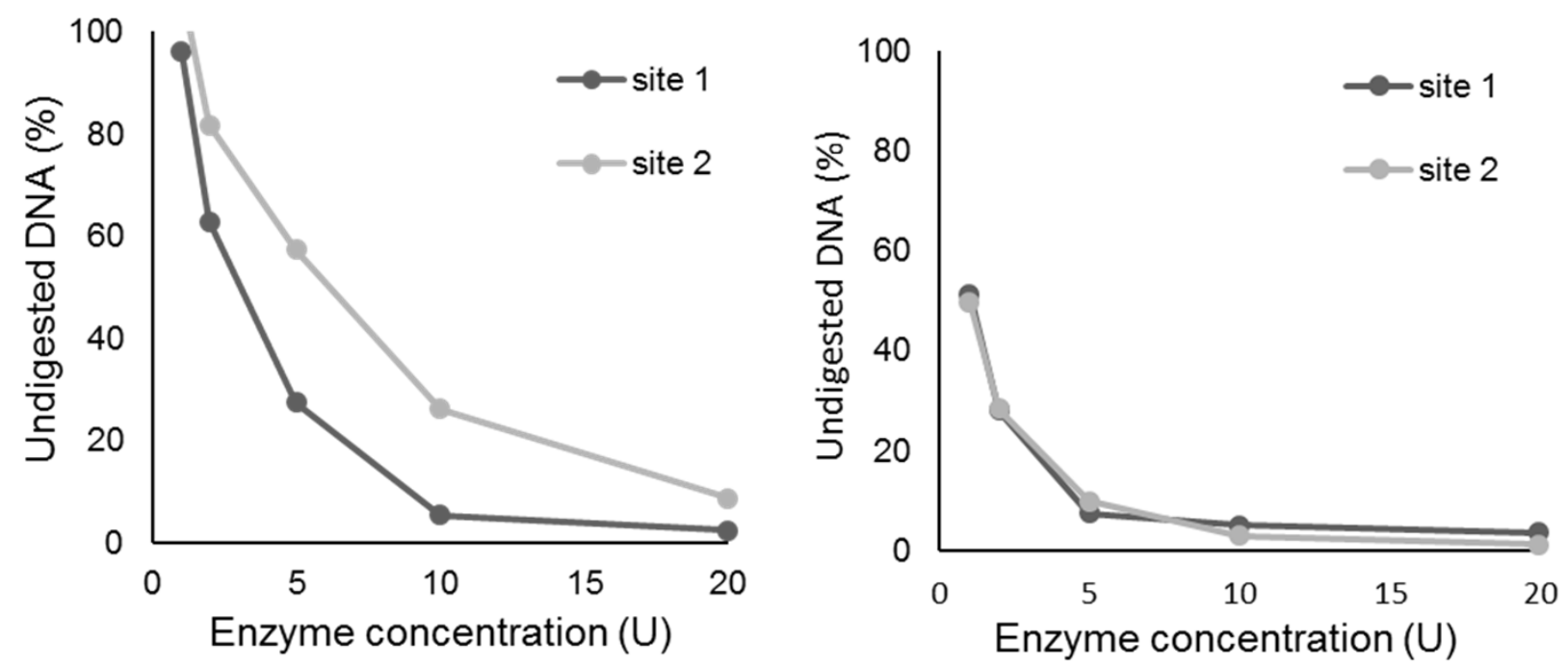

Figure 1 
A

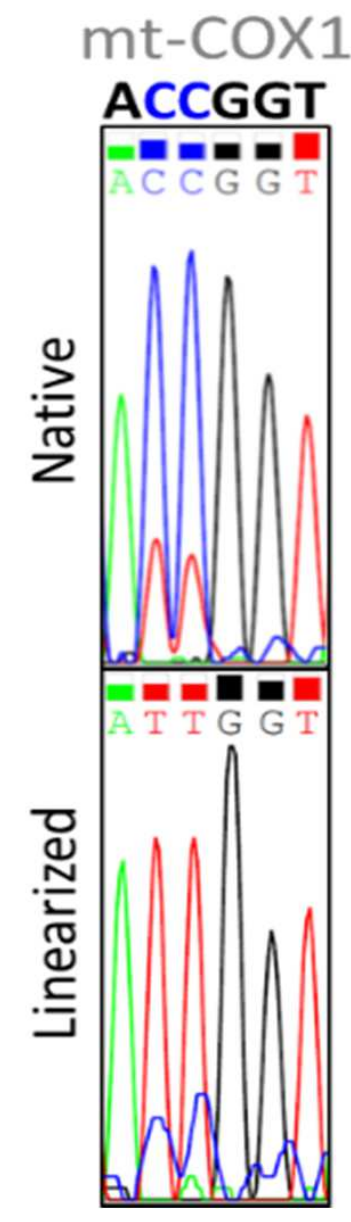

B

mt-Rnr1

CCGGTCT

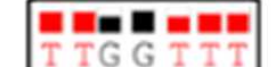

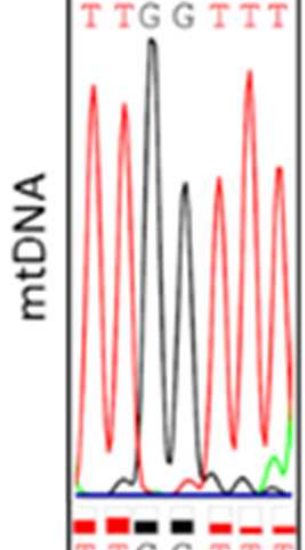

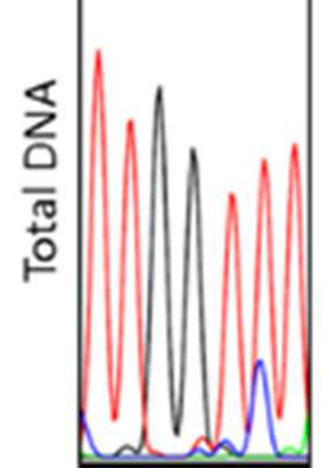

Figure 2 
A

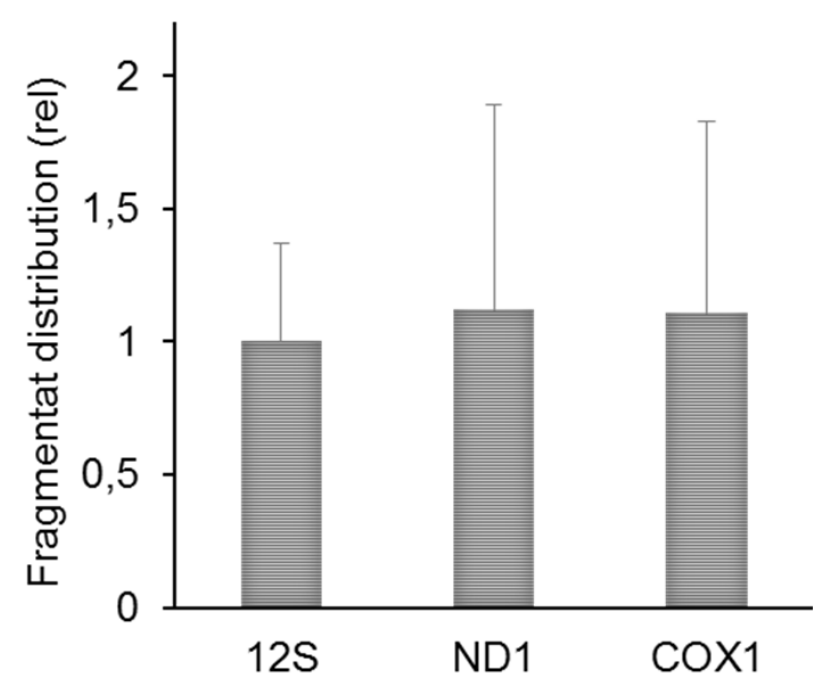

C

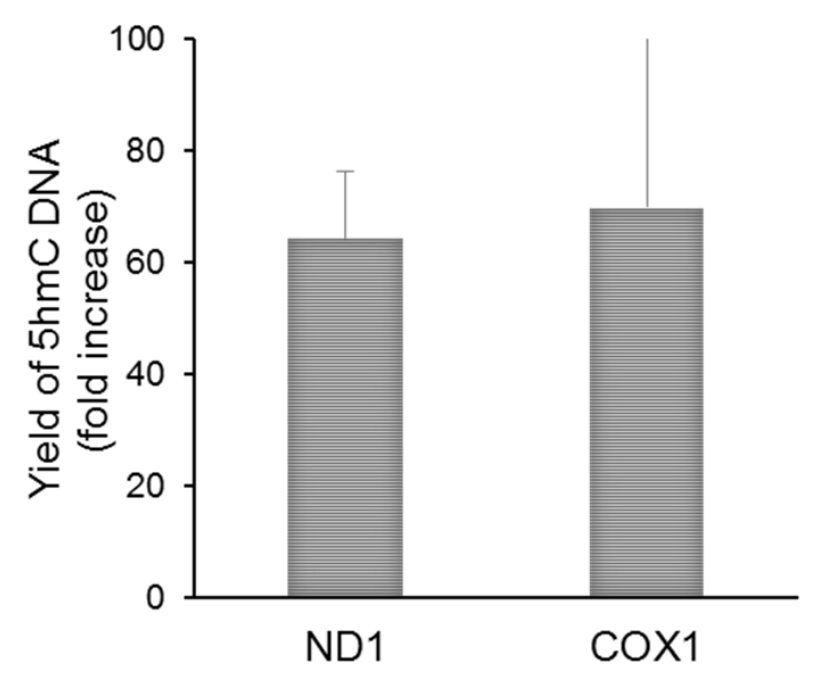

B

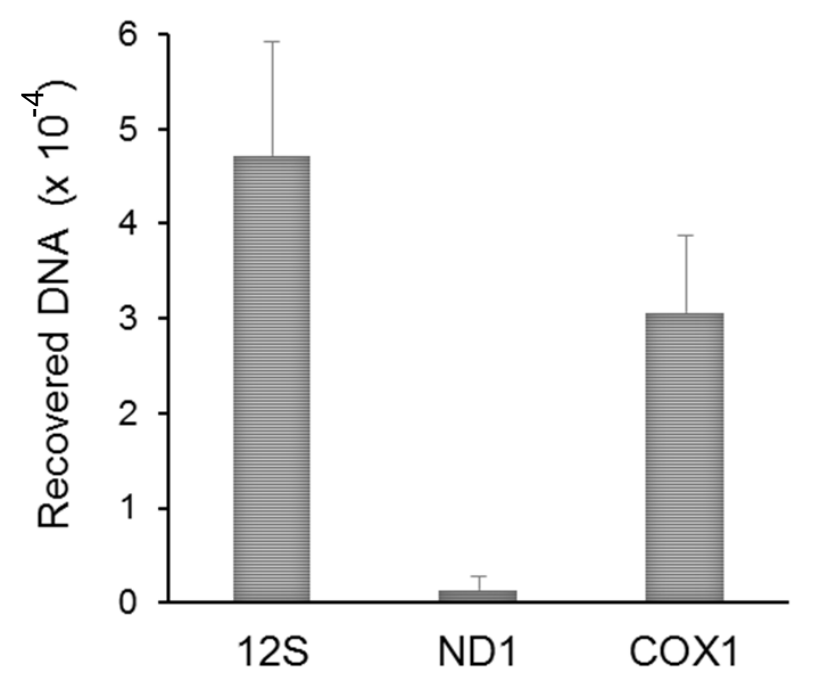

D

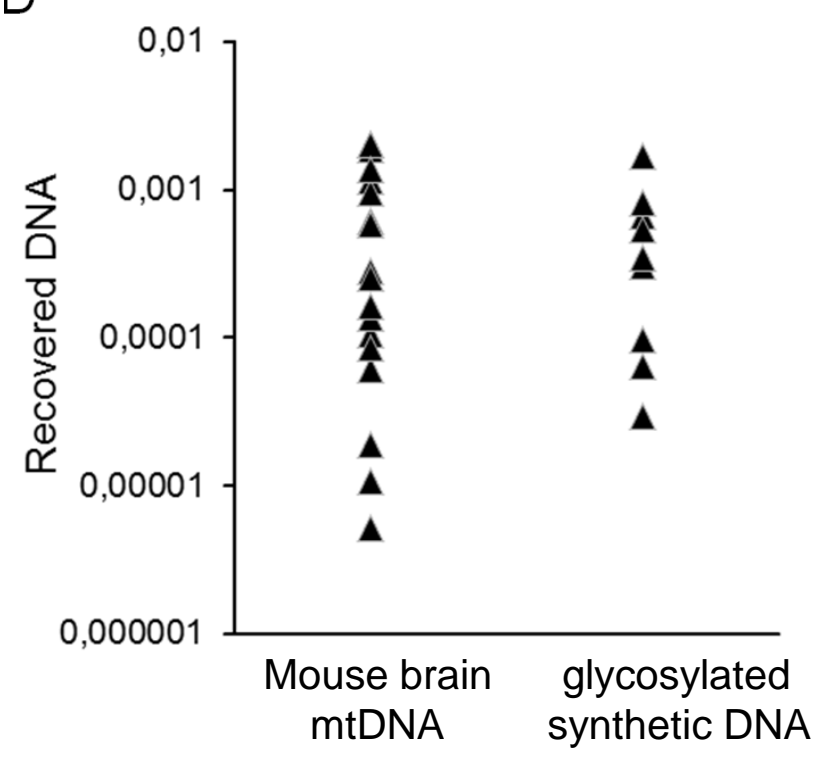

Figure 3 